\title{
Update on the Cytogenetics and Molecular Genetics of Chordoma
}

\author{
Lidia Larizza', Pietro Mortini' ${ }^{2}$ Paola Riva' \\ ${ }^{1}$ Department of Biology and Genetics for Medical Sciences, University of Milan, Italy; ${ }^{2}$ Department of Neurosurgery, University of Brescia, Italy
}

Key words: chordoma, 1p36 LOH, 7q33 linkage, TSC association

Corresponding author: Lidia Larizza, MD, Department of Biology and Genetics, Medical Faculty, University of Milan, Via Viotti 3/5 20133 Milan, Italy, phone +39 025031 5859, fax +39 025031 5864, e-mail: lidia.larizza@unimi.it

Submitted: 25 January 2005

Accepted: 7 February 2005

\begin{abstract}
Chordoma is a rare mesenchymal tumour of complex biology for which only histologic and immunohistochemical criteria have been defined, but no biomarkers predicting the clinical outcome and response to treatment have yet been recognised. We herein review the interdisciplinary information achieved by epidemiologists, neurosurgeons and basic scientists on chordoma, usually a sporadic tumour, which also includes a small fraction of familial cases. Main focus is on the current knowledge of the genetic alterations which might pinpoint candidate genes and molecular mechanisms shared by sporadic and familiar chordomas. Due to the scarcity of the investigated tumour specimens and the multiple chromosome abnormalities found in tumours with aberrant karyotypes, conventional cytogenetics and Fluorescence In Situ Hybridization failed to detect recurrent chordoma-specific chromosomal rearrangements. Genome-wide approaches such as Comparative Genomic Hybridization (CGH) are yet at an initial stage of application and should be implemented using BAC arrays either genome-wide or targeting selected genomic regions, disclosed by Loss of Heterozygosity $(\mathrm{LOH})$ studies. An $\mathrm{LOH}$ region was shown by a systematic study on a consistent number of chordomas to encompass 1 p36, a genomic interval where a candidate gene was suggested to reside. Despite the rarity of multiplex families with chordoma impaired linkage studies, a chordoma locus could be mapped to chromosome $7 \mathrm{q} 33$ by positive lod score in three independent families. The role in chordomagenesis of the Tuberous Sclerosis Complex (TSC) genes has been proved, but the extent of involvement of TSC1 and TSC2 oncosuppressors in chordoma remains to be assessed. In spite of the scarce knowledge on the genetics and molecular biology of chordoma, recent initiation of clinical trials using molecular-targeted therapy, should validate new molecular targets and predict the efficacy of a given therapy. Comparative genetic and biomolecular studies should enhance the molecular taxonomy of chordoma which might have a prognostic significance and better orient the therapeutic options.
\end{abstract}

\section{Epidemiology}

Chordomas are rare, low-to-intermediate grade malignant tumours which occur along the length of the craniospinal axis. They are attributed to neoplastic transformation of embryonic remnants of the primitive notochord [1]. Incidence is around 0.05/100,000/year
[2]. They account for $<1 \%$ of the central nervous system tumours [3] and $<5 \%$ of all primary malignant bone tumours [4]. The most common locations are the sacrococcygeal region (45-49\%), the base of the skull (36-39\%), and the spinal axis (8-15\%) [5]. In the cranio-cervical region seven points of origin have been indicated: dorsum sellae, Blumenbach's clivus, 
retropharyngeal notochord vestiges, remnants in the apical ligament of the dens, nuclei pulposi of the cervical vertebrae, vestiges in the squama occipitalis, and ectopic localizations [6]. Nose and paranasal sinuses primitive chordoma have been suggested not to be real ectopic localizations as more properly chordoma arose from remnants [7].

No racial predilection for chordomas has been reported, male-to-female ratio is generally 2 : $1[3,8]$. Every age can be involved with predominance for the third and fourth decades for intracranial localization, while spinal chordomas are generally observed at older ages because of late signs and symptoms [3,8]. Chordoma has no known association with irradiation or any other environmental factors. A small percentage of cases have a familial pattern of inheritance [9-13].

\section{Clinical presentation, therapy and prognosis}

Chordomas develop in the bone, so they initially grow extradurally with bone destruction and secondary extension into adjacent soft tissues [14]; they have common characteristics of a malignant tumour, with local invasiveness, tendency for recurrence, and the potential to metastasize. Distant metastases of chordomas have been described with prevalence up to $43 \%$ [15]; almost all cases were observed in sacrococcygeal localizations; the low rate of systemic spread for skull base neoplasms, which ranges from $0 \%$ to $10 \%$, is probably related to the fact that the patients die of the local effect of their tumours before metastases develop $[16,17]$.

Clinically they are slow-growing tumours characterized by local spread. Symptoms manifest late, even after years, and therefore the local extent of disease is often huge at diagnosis. Pain and neurological symptoms from local compression are the main subjective complaints of the patient. They obviously depend on tumour location. Symptoms like (from the earliest to the latest) low back pain, anaesthesia and paraesthesia, intestinal obstruction, are caused by sacral chordoma. Symptoms and signs of compression of nerve roots and/or spinal chord are related to vertebral chordoma.

Headache and diplopia are the most common symptoms related to chordoma of the clivus and base of the skull [18].

The $V$ cranial nerves are also frequently involved, due the progressive growth with invasion of other neighbouring structures such as the cavernous sinus. Signs and symptoms include also visual loss and limitation of visual field. Extraocular complaint can be dysphagia, dyspnea, dysphonia, facial pain, facial paresis, hearing loss, tinnitus, dizziness and ataxia, after brain stem compression. Anterior extension to the pharynx can explain pharyngolaryngeal and otological symptoms, whereas extension to nose and paranasal sinuses can cause nasal obstruction, ipo-anosmia, hyponasal speech, mucopurulent discharge, and, rarely, epistaxis $[5,8]$.

Distant metastases may occur late across the natural history of the disease, mainly to the lungs, but also to bone, liver, distant soft tissues and skin.

Given the natural history and histological characteristics of the disease, treatment is based on local modalities. Surgery is the treatment of choice. The aggressiveness of local resection has been correlated to the patient's outcome, in terms of both local control and survival. However, surgery is often confronted by major sequelae, which may follow adequate excision of several sacral presentation, with loss of urogenital and rectal function in case of bilateral section of S2, or below, nerve roots, as well as cranial nerves impairments after resection of clival chordomas.

Postoperative radiotherapy, both by linear accelerator, or proton therapy, can provide better control of the disease $[17,19,20]$.

Prognosis of chordomas is related to the extent of surgical removal: five-year survival of $35 \%$ is reported with incomplete resection also if followed by conventional radiation therapy [21]. Better results are reported with aggressive surgical treatment and proton-beam postoperative radiotherapy with a disease-free survival rate from $50 \%$ [22] to $77 \%$ at 5 years [20] and from $45 \%$ to $69 \%$ at 10 years $[19,20]$.

If surgery is unfeasible, radiation therapy is the second choice in order for eradicative intent. Chordoma is a relative radioresistant tumour, with 60 70 Gy of photon radiation therapy needed for best responses. Progression-free survival rates in the 30\% range have been reported with radiation therapy alone.

Chemotherapy has been resorted to, but only anecdotal reports of activity of chemotherapeutic agents or regimens are available. Drugs active in sarcomas, including Doxorubicin and Ifosfamide, as well as in carcinomas or other bone sarcomas, including Cisplatin and Etoposide, have been administered. However, chordomas are generally viewed as chemo-resistant, low-grade tumours, for which standard cytotoxic chemotherapy lacks an established role, even a palliative one, in advanced chordoma.

The prognosis of chordoma is affected by a variety of clinical and pathologic characteristics. Important features include tumour location, size and resectability, as well as the age and the gender of the patient; larger tumours, female gender, and age of over 40 years have been associated with a poorer outcome [16, 17, 23, 24]. 


\section{Macroscopic/microscopic features and immunohistochemistry}

Chordomas are generally whitish soft multilobulated mass with a fibrous pseudocapsule sometimes filled by a mucoid substance (secondary to haemorrhage), sometimes with haemorrhages, necrosis and/or calcifications and fragments of bone [1]. Microscopically, they are characterized by the vacuolated physaliphorous cells, which are translucent cells of different sizes rich in mucin and glycogen [1]. The mucin produced by the cells tends to collect outside them creating large nests of bubbly cells in a mucoid matrix. Other cells rest in chords anastomosing in the mucoid matrix. The nuclei are small and dark stained, indicating the minimally aggressive biologic behaviour. A representative histological section of a typical clival chordoma composed of nests and cords of tumour cells is shown in Fig. 1A, while the hallmark physaliphorous cells containing multiple clear cytoplasmic vacuoles can be seen in Fig. 1B.

Chordomas are of three overlapping and sometimes coexisting histopathologic types: conventional, chondroid and dedifferentiated. The chondroid chordoma, first described by Heffelfinger in 1973 [25], is characterized by a cartilaginous hyaline component and by a supposed better prognosis [26]. Dedifferentiated or sarcomatoid chordomas are high-grade neoplasms, which account for only $5 \%$ of cases [27].

Differential diagnosis of chordomas includes primary bone tumours, cartilagineous neoplasms such as chondromas or chondrosarcomas, epithelial neoplasms such as mucinous-forming adenocarcinoma or salivary neoplasms, metastases, neurinoma, neurofibroma, meningioma, neuroblastoma, haemangioma and lymphoma.

S-100 positivity, often seen in chordomas (Fig. 1C), can differentiate from epithelial neoplasms $[28,29]$. Cytokeratin antibodies and EMA (epithelial membrane antigen) positivity of chordoma (Figs. 1D and IE) is used to distinguish it from cartilaginous neoplasms, where the absence of these epithelial markers should be the rule [30]. Some chordomas stain positive with vimentin antisera (Fig. 1F), which reflects mesenchymal differentiation [31].

\section{Embryogenetic origin}

Chordoma is a disembryogenetic tumour attributed to malignant transformation of intraosseous persistent notochordal tissue [4]. In agreement with this view are the close histological and immunohistochemical similarities with the embryonic notochord and the correlation of chordoma development to location and incidence of notochordal vestiges [32].
It is known that in chick embryo the notochord develops through four periods of activity which are related to cytodifferentiation and functional maturation, with cessation of mitosis, cell apoptosis and decrease in the nucleolar volume in the fourth period [33]. It was also found by canine/bovine notochord cell cultures that a small number of notochordal cells persist in the nucleus pulposus with the function of maintaining disk integrity [34]. In human, the notochord forms from ectodermal cells during the third gestational week [32], then inducing chondrification and segmentation of the mesenchymal elements of the vertebral bodies. It obliterates in the second gestational month, leaving behind microscopic foci of notochord tissue in the most cranial and caudal of vertebral bodies. During the involution process the notochord normally completely disappears from the vertebrae to eventually form the nucleus pulposus of the intervertebral disk which is progressively replaced by fibrocartilage from the surrounding tissue by the age of 1-3 years. Macroscopic notochordal remnants, termed ecchordosis physaliphora (EP) are found at the base of the skull in up to $2 \%$ of adult autopsies and have been considered as one of the precursor lesions of classic chordomas [32, 35]. It has been pointed out that classic vertebral chordomas occur in bone and do not develop in the notochordal vestiges of the intervertebral disks [36]. In keeping with this prediction a few intraosseous benign notochordal cell tumours have been described [37, 38] and recently the first histologically confirmed case of a classic chordoma in the coccyx arising in a precursor benign notochordal lesion has been reported [39]. Intraosseous counterparts at the base of the skull have been also documented in a large microscopic study on vertebral columns from atlas to coccyx and pieces of the clival portion of the skull base dissected from 100 autopsy cases: a surprisingly high incidence (26 in 20 cases) of intraosseous benign notochordal cell tumours with anatomical distribution and immunohistochemical reactivity identical to that of classic chordomas have been identified [40]. The results showed that $11.5 \%$ of the clivus, $5.0 \%$ of the cervical vertebrae, $0 \%$ of the thoracic vertebrae, $2.0 \%$ of the lumbar vertebrae and $12 \%$ of the sacrococcygeal vertebrae were affected. These results support other evidence that classic chordomas develop from intraosseous benign notochordal cell tumours.

\section{Familial chordoma}

Chordomas are usually sporadic. However 8 families with multiple affected members have been reported (Table 1), suggesting genetic predisposition to chordoma [9, 10, 12, 41-45]. Three families showed a pattern of inheritance compatible with 
Table 1. Families with multiple occurrence of chordoma

\begin{tabular}{llll}
\hline Reference & Affected & Chordoma Site & Onset \\
\hline Foot et al, 1957 & brother and sister & sacrococcygeal & middle-age \\
\hline Enim et al, 1963 & two brothers & nasopharyngeal & youth \\
\hline Kerr et al, 1975 & 3 in three generations & nasopharyngeal & not specified \\
\hline Chetty et al, 1991 & 1 with family history & clival & 3 years \\
\hline Korczak et al, 1997 & 9 in three generations & clival, spinal & not specified \\
\hline Stepanek et al, 1998 & 4 in 2 generations & sacral, clival nasopharyngeal & 20, 39, 28, 31 \\
\hline $\begin{array}{l}\text { Dalprò et al, 1999 } \\
\text { Miozzo et al, 2000 }\end{array}$ & father and two daughters* & clival & before 20 \\
\hline Kelley et al, 2001 & two first cousins once removed & clival & not specified \\
\hline *hird with astrocytoma & & & \\
\hline
\end{tabular}

an autosomal dominant trait $[9,10,45]$. Genomic screen for linkage in the first of such families suggested possible linkage of the disease locus to a region on chromosome 1, 17 or 19, based both on parametric and non-parametric results [45], but no further data were released. Two complete linkage studies were performed on the other two families with autosomal dominant chordoma and putative susceptibility loci were mapped to $1 \mathrm{p} 36$ and $7 q 33[11,12]$, but so far the responsible genes have not been hunted from the target genomic regions. The study by Miozzo et al [1 1] concerned a male patient, who showed two recurrences of a clival chordoma developed when he was young, and his three daughters, two of whom expressed in infancy an astrocytoma and a chordoma, respectively. The eldest sibling was apparently healthy at the time of the investigation. We had previously reported a cytogenetic study on the chordoma recurrences from the index case showing extensively rearranged tumour karyotypes with chromosome $1 p$ involved in different unbalanced rearrangements leading to $1 p$ deletion [10]. This finding guided an $\mathrm{LOH}$ study coupled to segregation analysis of the 1 p36 region, in this two-generation family; results were consistent with the localization in 1 p36 of a tumour suppressor locus involved in chordoma predisposition [11]. Subsequent LOH studies in a cohort of 35 sporadic chordomas confirmed involvement of 1 p36 in the tumour development by $\mathrm{LOH}$ of region-specific polymorphic markers in the great majority of the tumours (31/35) [46 and unpublished observations], consistent with previous cytogenetic [47] and CGH [48] findings. Conversely, the linkage analysis is currently subjected to validation following the information that the eldest daughter, who was 17 years old at the time of our first reports $[10,11]$, subsequently developed a chordoma of the clivus. The patient was surgically treated elsewhere and we could not carry out additional studies. The previous segregation analysis evidenced sharing of the same haplotype at several 1 p36 loci among the father, the second and the third daughters, while the eldest daughter did not share it. We are currently reinvestigating by means of all available polymorphic markers the target region aiming at assessing whether a very small 1 p36 subregion segregating with genetic predisposition to chordoma might be disclosed.

The study performed by Kelley [12] in the family reported by Stepanek et al [9] consisted in a genomewide analysis for linkage which first yield a 2.2 lod score at marker D7S2195 on chromosome $7 \mathrm{q}$, based on only the 10 affected family members. To increase the power of the linkage analysis and to narrow the candidate disease gene region, additional members of this large family were examined together with two small independent families, one with two affected cousins, once removed, and another representing a branch of a previously reported threegeneration family [43]. The combined analysis with 20 markers on $7 q$ showed a maximum two-point lod score of 4.05 at marker D7S500. Multipoint analysis based only on the affected individuals and haplotyping of the three families members pinpointed a minimal disease region of $11.1 \mathrm{cM}$ from D7S1804 to D7S684 consistent with mapping to $7 q 33$ of a locus for familial chordoma. No $\mathrm{LOH}$ was found at any of the markers residing in the $7 q$ critical region, precluding narrowing of the candidate region [12]. 
So far, $7 q 33$ emerges as a strong candidate region for chordoma susceptibility based on the significant lod score obtained by the combined analysis of three families, one of which with a well suitable structure for linkage analysis. Conversely 1 p36 is a proven $\mathrm{LOH}$ region in chordoma [1 1, 46], which might harbour a susceptibility gene, should the linkage be reassessed in the family with two members showing $\mathrm{LOH}$ in the tumours and/or in additional families. Both $7 q 33$ and $1 p 36$ regions are compatible with the suspected heterogeneity of susceptibility loci for chordoma. Evidence for this is also provided by the outcome of chordoma in the context of Tuberous Sclerosis (MIM\#191100), an autosomal dominant syndrome, characterised among different clinical signs, by hamartomas in multiple organs, which is caused by germline mutations in either of two genes TSC1 (MIM 605284) and TSC2 (MIM 191092) which behave like tumour suppressors. TSC1 and TSC2 products, known as hamartin and tuberin, act as a heterodimer; thus the inactivation of either gene impairs the same pathway leading to the same clinical phenotype [49-51]. The association between chordoma and TSC has been highlighted by Lee-Jones et al [13] who datamined the literature for the unusual occurrence of chordoma in the context of tumour predisposition syndromes and identified three reports of chordomas in patients with Tuberous Sclerosis Complex (TSC) [52-54]. The same group demonstrated in two cases of sacrococcygeal chordomas developed by a newborn with germline TSCl mutation and a 33-week aborted foetus with a germline TSC2 mutation, somatic inactivation of the corresponding wild type allele by $\mathrm{LOH}$ and immunohistochemistry [13]. It should be noted that the early onset characterises also the three reported TSCassociated chordomas, which were discovered either during the first days of life [52] or in childhood [53,54] consistent with genetic predisposition mediated via germline mutation in a TSC gene.

No other cases of chordomas found with any coexisting tumours in the context of cancer predisposition syndromes are known.

\section{Genetic alterations in chordoma: cytogenetics, FISH and CGH}

The cytogenetic description by conventional banding and FISH-based techniques is restricted to less than 100 cases of chordoma worldwide. The percentage of tumours with normal karyotype was estimated on a sample of 18 chordomas to be slightly over $50 \%[55,56]$, but this figure is likely dependent on chordoma stage, anatomical site and type (primary or recurrent). The first report of a chordoma, which by

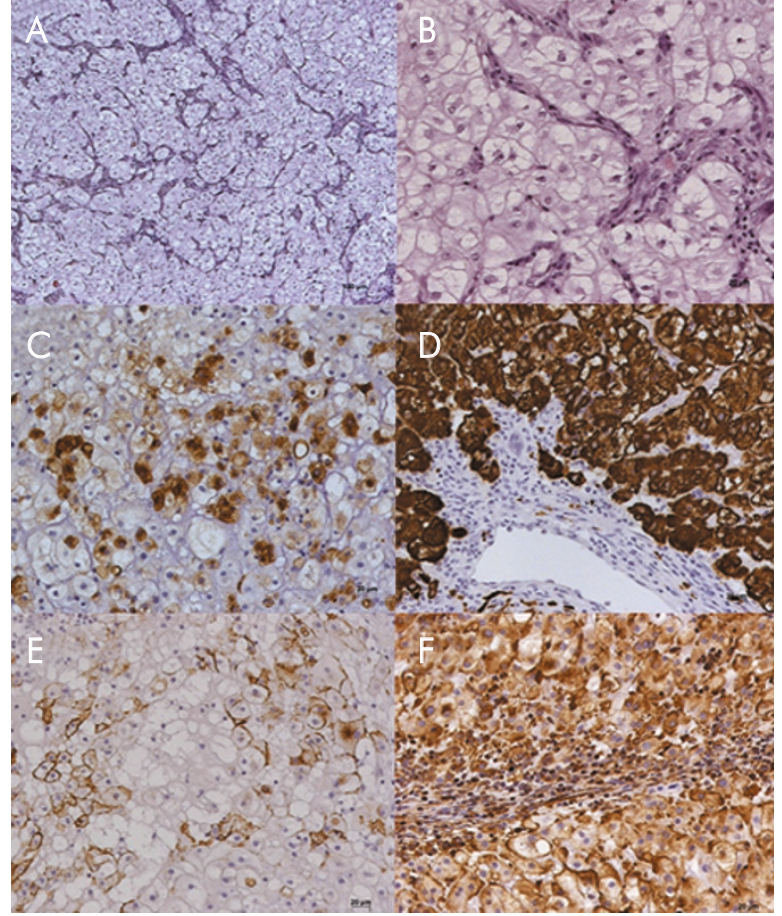

Fig. 1. Classic Chordoma

A: E-E, X 5; chordoma composed of nests and cords of tumour cells;

B: E-E, X 20; physaliphorous cells containing multiple clear cytoplasmic vacuoles; C: S-100, X 20; immunohistochemistry showing staining for S- 100 protein; D: CK, X 20; immunohistochemistry showing staining for cytokeratin; $E$ : EMA, X 20; immunohistochemistry showing staining for EMA;

F: Vimentin, X 20; immunohistochemistry showing staining for Vimentin

cytogenetic analysis showed two unrelated near-diploid clones with both numerical and structural abnormalities, was published by Persons et al [57]. Twenty-nine cases of conventional chordoma with aberrant karyotype are recorded in the Mitelman Catalog of Chromosome Aberrations in Cancer [CGAP] and six additional tumours, recently published [58,59], can be added to this list. Out of 35 chordomas with aberrant karyotype, 18 are annotated as recurrences: two as metastases, four as primary tumours, while no information is available for the remaining 11 tumours. The high rate of recurrent tumours among chordomas with aberrant karyotype is in keeping with the suggestion that in chordomas chromosome aberrations appear as late events in tumour progression [47]. Chondroid chordomas, i.e. chordomas containing foci of cartilage, that are morphologically indistinguishable from chondrosarcomas, are not considered here as it is controversial whether they represent a variant of conventional chordoma. Also dedifferentiated chordoma, of which only one case has been cytogenetically characterised and found to have a polyploid complex karyotype with double minutes 


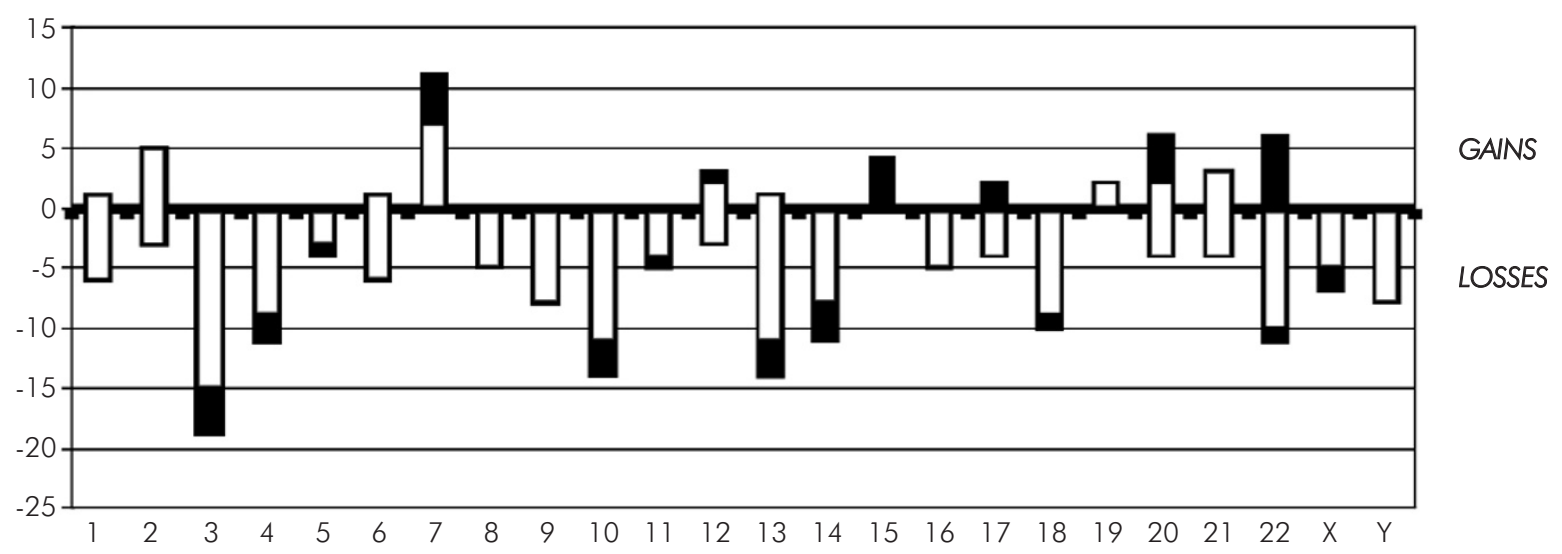

Fig. 2. Histogram showing the absolute number of chordomas $(n=57)$ with the respective gain or loss of entire chromosomes plotted for the whole genome. The white bars refer to conventional cytogenetics data [CGAP and 58], while the black bars refer to CGH data [48 and 65 ]. Chromosomes are arranged along the abscissa ( 1 to $22, X$ and $Y$ )

[59] is not included, as its relation with simple chordoma is unknown. Out of 35 karyotyped conventional chordomas with aberrant karyotype, the great majority are either hypo or quasi-diploid and only two are hyperdiploid [47, 60]. Only a few cases have simple karyotypes with one or more aberrations, but no underlying breakpoint was shared among chordomas characterised by a sole balanced translocation or rearrangement $[47,61-64]$. So far there are no clues that specific translocations, resulting in chimeric genes, play a pathogenetic role in chordomas. Most tumours have both numerical and structural chromosomal aberrations, and those with a complex karyotype often display clonal heterogeneity. As to numerical chromosome abnormalities, losses involve in the order chromosomes 3, 13, 10, 22, 4, $18,14,9$ and $Y$, while gains affect preferentially chromosomes 7, 2 and 21 [CGAP]. Imbalances of chromosome arms or subchromosomal regions were also identified, most commonly loss of $3 p, 3 q$ or both segments, loss of $1 p$ and $9 p$, but in most cases these were gross alterations, and their pattern appears to be non specific [CGAP]. A large variety of rearrangements, among which isochromosome la and loss of part or all of $1 p$ were identified as equivalent recurrent changes associated with chordoma progression [10, 47]. Loss of 1 pter-p34 has been detected in 14 cases [CGAP, 58] suggesting that a tumour suppressor locus might map in 1 p36 [11]. Other recurrent structural changes were add (21) (q22) and del (X) (p22), but none of them appeared to be tumour specific. Sixteen chordomas were surveyed by CGH and FISH [48] and six additional ones were preliminary investigated by the same approach [65]. Cumulative data obtained on the imbalance of entire chromosomes are combined to previous findings obtained by conventional cytogenetics to depict the overall profile of chromosomes gained and lost in an overall series of 57 chordomas (Fig. 2). CGH analysis also evidenced copy number changes restricted to chromosome arms or smaller genomic regions, but due to the limited number of tumour samples, only a few generalities can be drawn. One, at apparent discordance with karyotypic data, is that gains were more common than losses. One third of the tumours analysed by $\mathrm{CGH}$ combined to FISH were hyperdiploid: a finding making the authors suggest that hypo or near diploid cell populations have a growth advantage in vitro [48]. Hypodiploidy is not a feature of human solid tumours. DNA-flow cytometry data also suggested that hypodiploidy is not a common feature of chordoma either [66]. More precisely, the investigation of 16 chordomas showed that there was a median of six chromosomal imbalances per tumour, on average, 3.2 losses and 4.2 gains. Most common losses mapped on chromosomal arms $3 p(50 \%)$ and $1 p(44 \%)$ and most common gains involved $7 q(69 \%)$, $20(50 \%), 5 q(38 \%)$ and $12 q(38 \%)$ [48]. The consensus region for gains on chromosome $7 q$ was $7 q 36$, where the homeobox gene HLXB9 and sonic hedgehog gene $\mathrm{SHH}$ reside: interestingly both genes are a plausible candidate as they are expressed throughout the notochord during embryogenesis [67]. Another preliminary report on a panel of six cases confirms gains of chromosomal areas of $7 q$, but also discloses gains of $7 p, 1 q 23-24$ and 19p13 and, in agreement with earlier cytogenetic and FISH data, loss of 9p22-23 [65]. Use of high resolution FISH 

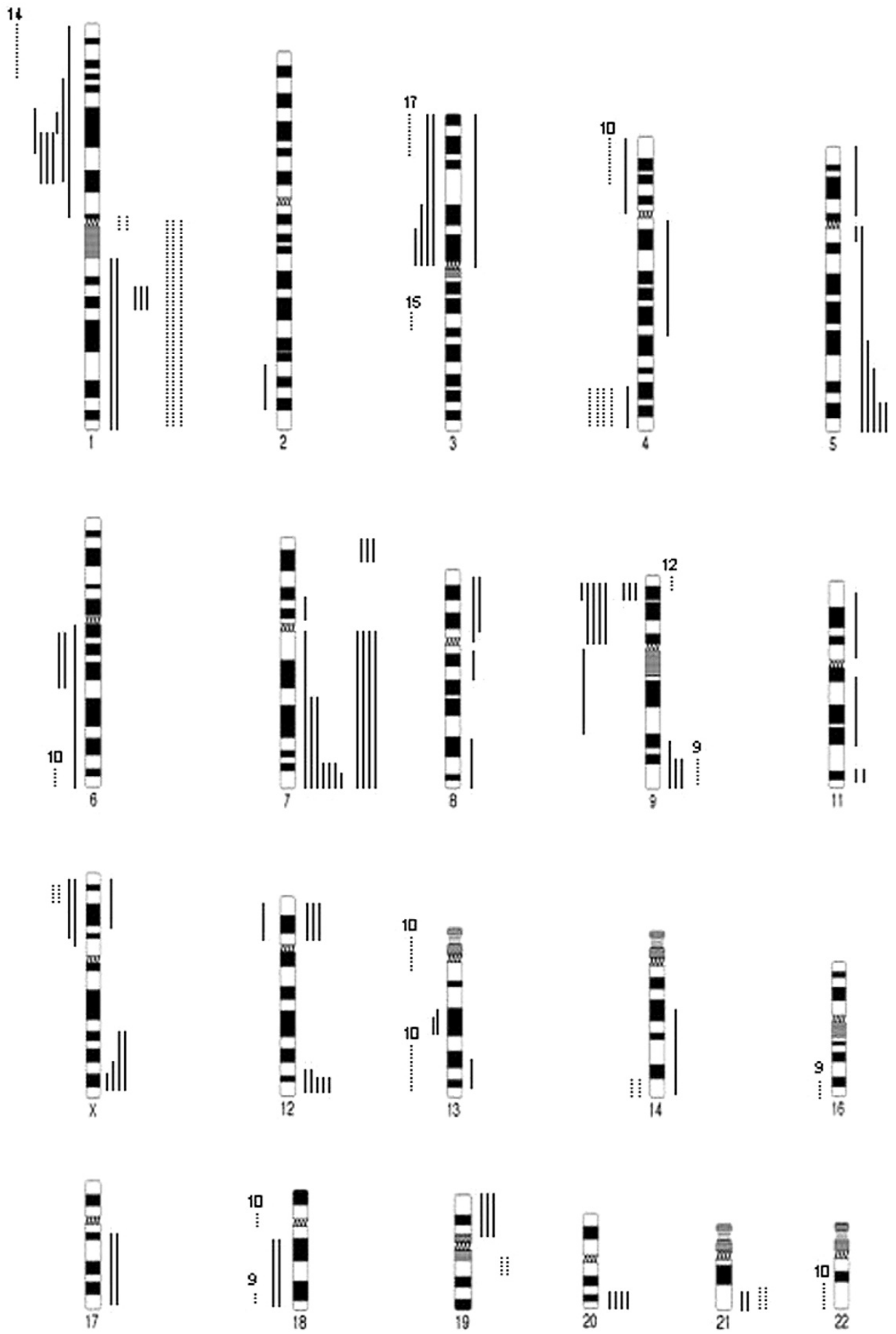

Fig. 3. Summary of copy number changes (except whole chromosome gains and losses) detected by cytogenetics [CGAP and 58] and CGH [48 and 65] in chordoma. Vertical lines on the left side of the chromosomes indicate losses and vertical lines on the right correspond to gains. Bold lines refer to CGH data, and dotted lines to cytogenetics findings. Each line corresponds to a tumour: when more than 4 tumours have the same aberration, the number is given above each line. Chromosomes 10,15 and $\mathrm{Y}$ are not included as no subchromosomal gains and losses were detected 
techniques, such as COBRA did not pinpoint any clustering of breakpoints, but confirmed loss of chromosome arms 1p, 3p, 3q, 9p and chromosome 10 [58]. Figure 3 shows a synoptic view of all the gross and subtle imbalances detected by conventional cytogenetics and $\mathrm{FISH}$, as well as by $\mathrm{CGH}$ in chordomas. Gains and losses are indicated by lines to the right and to the left of each chromosome ideogram. The distribution of copy number changes is here composed by two approaches, which should complement each other as the first one, the low-resolution banding karyotyping allows to disclose in heterogeneous tumours even low frequency clonal aberrations, which might be underscored by the high resolution whole genome $\mathrm{CGH}$ approach. However, the genomic imbalances evidenced by these matched tools (loss of 1p, 3p, 4p, 6q, 18q and Xp and gain of $1 q, 9 q$, and $21 q$ ) affect gross areas which should be narrowed by other most powerful approaches, namely $\mathrm{CGH}$ by means of full coverage BAC arrays and region-specific $\mathrm{LOH}$ studies. In addition, the number of chordomas analysed so far remains too small to drive general insights. This constraint and the relatively unspecific location of recurrent band imbalances and breakpoints make their pathogenetic significance elusive. The mapping of candidate genes in chordomagenesis awaits the application of high resolution targeted approaches.

\section{Genetic alterations in chordoma: LOH studies}

The first $\mathrm{LOH}$ study on chordomas concerned the $\mathrm{Rb}$ locus (13q14) at which $\mathrm{LOH}$ was detected in two of 7 sphenooccipital/clivus tumours, and proposed by correlation with the clinical behaviour as a marker of aggressive tumours [68]. The finding is consistent with the loss of chromosome 13 (most frequently occurring after chromosome 3) ascertained by cytogenetics and CGH [CGAP; 48]. Further LOH studies evidenced the loss of 17p, 9p and 18q, where known oncosuppressor genes are mapped [69]. It has been reported that the combined loss of p53 function and RB 1 protein leads to genomic instability, a finding consistent with the model of progressive accumulation of genetic changes with increasing malignancy $[68,70]$.

A targeted study involved the 1 p36.13 interval [46], which is comprised within the commonly deleted chromosome $1 p$ in chordoma $[10,47,58,60]$. The 1 p36.13 band had been pinpointed by the recurrent breakpoints identified in two tumour recurrences of the founder of an Italian chordoma family [10] and the haplotype and $\mathrm{LOH}$ information retrieved on this family
[11]. Typing of 31 region-specific microsatellites evidenced $\mathrm{LOH}$ across 1 p36.13 in 25 out of 27 sporadic chordomas which were tested [46], data confirmed by further analyses on a wider tumour panel (unpublished observations). A common deleted region, with a genetic length of $3.9 \mathrm{cM}$, was shared by 23 chordomas, raising the option of hunting candidate genes in this region. A few difficulties are represented by the consistent physical length (3 Mb) of this subtelomeric region, the high gene density and its common loss in a wide spectrum of solid tumours, mainly neurological [71, 72], suggesting a possible non-specific role in chordoma. The first selection of region-specific genes was based on genes with functions related to development or regression of the notochord such as Caspase 9 (CASP9) and Ephrin 2A (EPH2A). CASP9 is a ubiquitously expressed protease which triggers the apoptotic pathway by releasing cytochrome c from mitochondria into the cytosol [73]. $\mathrm{EPH} 2 \mathrm{~A}$ is a tyrosine kinase receptor involved in tail notochord formation during mouse embryo development [74]. The murine orthologue is regulated by members of the p53 gene family and plays an important role in apoptosis: it is found upregulated during angiogenesis in tumours [75]. Additional candidate genes come to the attention when a wider $\mathrm{LOH}$ region, which is shared by a lower percentage (40\%) of chordomas is considered. They include the paired box 7 (PAX7) gene encoding a transcriptional factor expressed in the neural tube which is regulated by notochord specific signals [76], the differentially screening-selected gene aberrant in neuroblastoma (DAN), involved in the negative regulation of cell proliferation [77], the Dishevelled 1 gene (DVL1), a key factor in Wnt signalling expressed in the neural tube [78] and a few genes belonging to the tumour necrosis factor receptor superfamily (TNFRSF-1B, -8, -9, - 14), the DNA fragmentation factor (DFF-A and-B) and TP73 [UCSC], all acting in apoptotic pathways.

Preliminary data on RT-PCR expression analysis of eight chordomas evidenced the lack of CASP9, EPHA2 and DVL 1 transcripts in 5, 1 and 4 tumours, respectively. Interestingly, some of the non-expressing tumours did not show 1 p36 LOH suggesting a loss of function as a result of point mutation or other mechanisms [46]. Because chordoma cells are unique, a control cell type of similar origin is difficult to identify; the nucleus pulposus is currently being used as suitable reference for expression studies.

No LOH has been detected in tumour specimens from the affected family members at 7q33, a region cosegregating with susceptibility to chordoma in the family described by Kelley et al [12]. According to the 
authors, the absence of $\mathrm{LOH}$ may indicate that the disease gene exerts its oncogenic effect in a dominant way. No $\mathrm{LOH}$ at 7q33 was detected in the tumours from the affected members of the Italian family [unpublished observations].

As reported above, the somatic loss of the wild-type TSC 1 and TSC2 allele was found in the chordomas developed by two patients with Tuberous Sclerosis, carrying germline mutations of either TSC gene [13]. TSC 1 and TSC2 are usually considered as a complex with one function. Genetic studies in mammalian systems [79] and Drosophila [80, 81] showed that the TSC1/TSC2 complex inhibits cell growth in both mass or size and cellular proliferation thus exerting a positive control of apoptosis $[82,83]$. It would be worthwhile monitoring $\mathrm{LOH}$ at both TSC genes in a significant sample of chordomas, especially sacrococcygeal chordomas which are ascertained in children [84] and might be on a hereditary basis, and proceed with the mutation screening in the $\mathrm{LOH}$-positive cases. The TSC genes, whose pathogenetic role in chordoma development has been disclosed, may provide through their impaired function a link to other susceptibility genes, yet to be detected.

\section{Molecular markers in relationship to clinical parameters}

There is still a deep gap in our understanding of the genetic basis and molecular biology of chordoma. Two studies have examined the role of molecular markers in chordoma in relationship to clinical parameters [56, 69], but currently they have only an exploratory meaning, which cannot lead to advance the care and management of this cancer.

The first study showed that chordoma cells from five patients had an increased telomere length compared with leukocytes from age-matched controls, in marked contrast to telomere length reduction which is observed in most cancers. Telomerase activity was present in chordoma cells from one of the two patients who were studied, but to a lesser degree compared with Hela [56]. The second study showed that chordomas can be added to the list of malignancies demonstrating microsatellite instability (MIM) which was evidenced in six out of 12 tumours tested, but pointed out that $\mathrm{LOH}$ may prove to portend a worse prognosis than MIN [69].

Chordoma is unique among mesenchymal tumours in the epithelioid features seen. Immunoreactivity for cytokeratins (Fig. 1D), epithelial membrane antigen (Fig. 1E), S-100 protein (Fig. 1C), vimentin (Fig. 1F) and neurofilaments helps diagnosis and discrimination from other mucin-producing bone cancers, but does not represent a prognostic feature. The complex immunophenotype of chordoma has been related to its origin from notochord, which undergoes conspicuous changes in location and morphology during embryonic development [85]. Detailed analyses were thus conducted on chordoma and foetal notochord aiming at studying the expression of each component of cytokeratin [86]. Cytokeratins CK8 and CK9 were found to be shared by both chordoma and notochord, as well as galectin-3, an endogenous carbohydrate-binding protein [87]. Recently galectin-3 has been demonstrated to be an immunohistochemical marker most useful to distinguish the pathologically overlapping entities of chordomas and myxoid chondrosarcoma [88].

The expression of cell adhesion molecules (CAMs) including E-cadherin, alpha-catenin, beta-catenin, gamma-catenin and neural cell adhesion molecule (NCAM) has been associated with formation and maintenance of chordoma tissue architecture and found of diagnostic value for discriminating chordoma from chondrosarcoma, along with the cytokeratins [89, 90]. However no significant correlation was found between the decreased expression of CAMs, observed in most chordomas and the histological grade of malignancy, cellular growth pattern or clinical parameters. Further studies tested the hypothesis that the expression of certain growth factors and/or structural proteins might be correlated with the biological behaviour of chordomas. Investigations on steroid hormone receptors, which are involved in tumour growth, evidenced that progesterone receptor $B$ and oestrogen receptor alpha were expressed in chordoma and hence associated with tumour progression [91]. High levels of transforming growth factor alpha and basic fibroblast growth factor expression were linked to higher rates of recurrence and strong fibronectin expression was also associated with poor prognosis, being thus considered an additional marker of aggressiveness [92].

\section{Conclusions and future directions}

Chordoma is a peculiar tumour, which constitutes a nosological entity by its own. Due to its rare occurrence the tumour specimens which could be processed for conventional and FISH-based chromosomal analyses are yet scarce. When the karyotype was aberrant, a wide variety of both numerical and structural aberrations had been detailed, but no distinctive sequence of aneuploidy or common tumour-specific chromosome rearrangements could be identified. Only one study based on standard $\mathrm{CGH}$ analysis has been applied and revealed a good 
matching with the previous cytogenetic findings [48] Analysis of additional tumour specimens is warranted to select common regions of imbalance for more detailed studies using array-CGH by full coverage of specific BACs and targeted $\mathrm{LOH}$. Positional candidate genes should thus emerge, besides those residing in genomic intervals, such as 1 p36 and 7 q33 which are thought to be relevant for chordoma genesis and/or progression and are currently under intense investigation. It is currently likely that a gene at 7q33 is involved in susceptibility to chordoma, according to the consistent linkage results obtained in 3 out of the 8 families with chordoma so far studied [12]. The lack of $\mathrm{LOH}$ at 7q33 markers, if corroborated by further data, would classify the predisposition gene as an oncogene, consistent with the common gain of $7 q$ detected by cytogenetics and molecular cytogenetics [48]. It is also well proven that the 1 p36 interval is preferentially lost in chordoma [46] and might thus harbour at least one oncosuppressor whose role in early or late stages of chordomagenesis should be pointed out. Whether or not germline mutation in this latter potential candidate might also sustain genetic susceptibility awaits confirmation. The role of TSC 1 and TSC2 genes in congenital or early onset chordomas has been established [13]. LOH test at both TSC genes should be a useful adjunct in chordomas to screen the positive cases for germline mutations allowing to establish the epidemiological contribution of these two cancer genes to chordoma onset. Position-independent candidate genes might also emerge by other approaches: the rationale behind it is their involvement in apoptotic pathways, as their constitutional or somatic deregulation might lead to defective notochord regression, i.e. the premise for subsequent neoplastic transformation. Once the pathogenesis of chordoma is elucidated, cytological markers of prognostic significance might be used in clinical practice. Therapeutic strategies could benefit from these discoveries. Recently imatinib mesylate (Gleevec, Novartis Pharma AG, Basel, Switzerland) [93], a highly selective inhibitor of the protein tyrosine kinase family that comprises $\mathrm{Abl}$, the platelet-derived growth factor receptor (PDGF-R) $\alpha$ and $\beta$ [94] and the product of the c-kit protooncogene, KIT, has been found to have anti-tumour activity in patients with advanced chordomas [95]. All the six patients who were treated had tumours found positive for PDGFR $\beta$ which was shown to be phosphorylated in four of them. Further investigation on the role of PDGFR $\beta$ in chordomas should substantiate these findings, allowing to prospectively register all those patients who might benefit from imatinib mesylate treatment.

\section{Acknowledgements}

The authors thank all those who took part in this study. We would like to acknowledge the following: Prof. AM Fuhrman Conti and L. Dalprà (University of Milano Bicocca), Dr M. Miozzo, Dr F. Orzan and Dr M. Longoni (University of Milan), Prof. C. Doglioni and Dr N. Boari (S. Raffaele Hospital, Milan). This work was partially supported by an AIRC (Associazione Italiana per la Ricerca sul Cancro) 2001-2003 grant to L.L.

\section{Electronic-database information}

The accession number and URLs for data in this article are as follows:

- Online Mendelian Inheritance in Man (OMIM), http://www.ncbi.nln.nih.gov/Omim/for chordoma (OMIM 215400), Tuberous Sclerosis (OMIM \# 191 100), TSC1 (OMIM 605284) and TSC2 (OMIM 191092),

- Mitelman database of chromosome aberrations in cancer. Updated May 2003,

http://cgap.nci.nih.gov/chromosomes/Mitelman, Mitelman F, Johansson B, Mertens F, editors, recurrent Case for Diagnosis/chordoma,

- UCSC browser information,

http://www.genome.ucsc.edu, for TNFRSF-1B, -8, -9, - 14, DFF-A and -B and TP73.

\section{References}

1. Batsakis JG. Tumors of the Head and Neck. Williams and Wilkins, Baltimore, 1979.

2. McMaster ML, Goldstein AM, Bromley CM, Ishibe N and Parry DM. Chordoma: incidence and survival patterns in the United States, 1973-1995. Cancer Causes Control 2001; 12: 1-11.

3. Weber AL, Brown EW, Hug EB and Liebsch NJ. Cartilaginous tumors and chordomas of the cranial base. Otolaryngol Clin North Am 1995; 28: 453-471.

4. Mirra JM, Nelson SD, Della Rocca C and Mertens F. Chordoma. In: Fletcher CDM, Unni KK and Mertens F. editors. Pathology and Genetics of Tumors of Soft Tissue and Bone. World Health Organization Classification of Tumors. Lyon: IARC press 2002, pp 315-317.

5. Harrison DF and Lund VJ. Tumours of the Upper Jaw. Churchill Livingstone, Edinburgh, 1995.

6. Binkhorst CD, Schierbeek P and Petten GJW. Neoplasms of the notochord. Acta Otolaryngol 1957; 47: 10-20.

7. Shugar JM, Som PM, Krespi YP, Arnold LM and Som ML. Primary chordoma of the maxillary sinus. Laryngoscope 1980; 90: 1825-1830.

8. Perzin KH and Pushparaj N. Nonepithelial tumors of the nasal cavity, paranasal sinuses, and nasopharynx. A clinicopathologic study. XIV: Chordomas. Cancer 1986; 57: 784-796.

9. Stepanek J, Cataldo SA, Ebersold MJ, Lindor NM, Jenkins RB, Unni K, Weinshenker BG and Rubenstein RL. Familial chordoma with probable autosomal dominant inheritance. Am J Med Genet 1998; 75: 335-336.

10. Dalprà L, Malgara R, Mozzo M, Riva P, Volonte M, Larizza L and Fuhrman Conti AM. First cytogenetic study of a recurrent familial chordoma of the clivus. Int J Cancer 1999; 81 (1): 24-30. 
11. Miozzo M, Dalprà L, Riva P, Volonté M, Macciardi F., Pericotti S, Tibiletti MG, Cerati M, Rhode K, Larizza L and Fuhrman Conti AM. A tumor suppressor locus in familial and sporadic chordoma maps to 1p36. Int J Cancer 2000; 7 (1): 68-72.

12. Kelley MJ, Korczak JF, Sheridan E, Yang X, Goldstein AM and Parry DM. Familial chordoma, a tumor of notochordal remnants, is linked to chromosome 7q33. Am J Hum Genet 2001; 69: 454-460.

13. Lee-Jones L, Aligianis I, Davies PA, Puga A, Farndon PA, Stemmer-Rachamomov A, Ramesh $V$ and Sampson JR. Sacrococcygeal chordomas in patients with Tuberous Sclerosis Complex show somatic loss of TSC1 or TSC2. Genes Chrom Cancer 2004; 41: 80-85.

14. Oikawa S, Kyoshima K, Goto T, Iwashita T, Takizawa T, Kobayashi $S$ and Ito M. Histological study on local invasiveness of clival chordoma. Case report of autopsy. Acta Neurochir (Wien) 2001; 143: 1065-1069.

15. Higinbotham NL, Phillips RF, Farr HW and Hustu $\mathrm{HO}$ Chordoma: thirty-five year study at the Memorial Hospital. Cancer 1967; 20: 1841-1850.

16. Gay E, Sekhar LN, Rubinstein E, Wright DC, Sen C, Janecka IP and Snyderman $\mathrm{CH}$. Chordomas and chondrosarcomas of the cranial base: results and follow-up of 60 patients. Neurosurgery 1995; 36: 887-897

17. Hug EB, Loredo LN, Slater JD, DeVries A, Grove RI, Schaefer RA, Rosenberg AE and Slater JM. Proton radiation therapy for chordomas and chondrosarcomas of the skull base. J Neurosurg 1999; 91 : 432-439

18. Volpe NJ, Liebsch NJ, Munzenrider JE and Lessell S Neuro-ophthalmologic findings in chordoma and chondrosarcoma of the skull base. Am J Ophthalmol 1993; 115: 97-104.

19. Rosenberg AE, Nielsen GP, Keel SB, Renard LG, Fitzek MM, Munzenrider JE and Liebsch NJ. Chondrosarcoma of the base of the skull: a clinicopathologic study of 200 cases with emphasis on its distinction from chordoma. Am J Surg Pathol 1999; 23 : 1370-1378

20. Crockard HA, Steel T, Plowman N, Singh A, Crossman J, Revesz T, Holton JL and Cheeseman A. A multidisciplinary team approach to skull base chordomas. J Neurosurg 2001; 95: 175-183.

21. Zorlu F, Gurkaynak M, Yildiz F, Oge K and Atahan IL. Conventional external radiotherapy in the management of clivus chordomas with overt residual disease. Neurol Sci 2000; 21 203-207.

22. Colli B and Al-Mefty O. Chordomas of the craniocervical junction: follow-up review and prognostic factors. J Neurosurg 2001; 95: 933-943.

23. Forsith PA, Cascino TL, Shaw EG, Scheithaver BW, O'Fallon JR, Dozier JC and Piepgras DG. Intracranial chordomas: a clinicopathological and prognostic study of 51 cases. J Neurosurg 1993; 78: 741-747.

24. O'Connell JX, Renard LG and Liebsch NJ. Base of skull chordoma: a correlative study of histologic and clinical features of 62 cases. Cancer 1994; 74: 2261-2267.

25. Heffelfinger MJ, Dahlin DC, MacCarty CS and Beabout JW. Chordomas and cartilagineous tumors at the skull base. Cancer 1973; 32: 410-420.

26. Moriki T, Takahashi T, Wada M, Ueda S, Ichien M and Miyazaki E. Chondroid chordoma: fine-needle aspiration cytology with histopathological, immunohistochemical, and ultrastructural study of two cases. Diagn Cytopathol 1999; 21 : 335-339.

27. Saito A, Hasegawa T, Shimoda T, Toda G, Hirobashi S, Tajima $G$ and Moriya Y. Dedifferentiated chordoma: a case report. Jpn J Clin Oncol 1998; 28: 766-771.

28. Bottles K and Beckstead JH. Enzyme histochemical characterization of chordomas. Am J Surg Pathol 1984; 8: 443-447.
29. Walker WP, Landas SK, Bromley CM and Sturm MT. Immunohistochemical distinction of classic and chondroid chordomas. Mod Pathol 1991; 4: 661-666.

30. Wojino KJ, Hruban RH, Garin-Chesa P and Huvos AG. Chondroid chordomas and low grade chondrosarcomas of the craniospinal axis. Am J Surg Pathol 1992; 16: 1144-1152.

31. Bouropoulou V, Bosse A, Roessner A, Vollmer E, Edel G, Wuisman $P$ and Harle A. Immunohistochemical investigation of chordomas: histogenetic and differential diagnostic aspects. Current Topics in Pathology 1989; 80: 183-203.

32. Salisbury JR. Embryology and pathology of the human notochord. Ann Pathol 2001; 21 : 479-488.

33. Zavala G and Vazquez-Nin GH. Analysis of nuclear ribonucleoproteic structures during notochordal cell differentiation and maturation in chick embryos. Anat Rec 2000; 259: 113-123.

34. Aguiar DJ, Johnson SL and Oegema TR. Notochordal cells interact with nucleus pulposus cells: regulation of proteoglycan synthesis. Exp Cell Res 1999 Jan 10; 246 (1): 129-137.

35. Ulich TR and Mirra JM. Ecchordosis physaliphora vertebralis. Clin Orthop 1982; 163: 282-289.

36. Jaffe HL. Tumors and Tumorous Conditions of the Bone and Joints. Philadelphia: Lea and Febiger, 1958; pp. 451-461.

37. Darby AJ, Cassar-Pullicino VN, McCall IW and Jaffray DC. Vertebral intra-osseous chordoma or giant notochordal rest? Skeletal Radiol 1999; 28: 342-346.

38. Kryakos M, Totty WG and Lenke LG. Giant vertebral notochordal rest. Am J Surg Pathol 2003; 27 (3): 396-406

39. Yamaguchi T, Yamato M and Saotome K. First histologically confirmed case of a classic chordoma arising in a precursor benign notochordal lesion: differential diagnosis of benign and malignant notochordal lesions. Skeletal Radiol 2002; 31: 413-418.

40. Yamaguchi T, Suzuki S, Ishiiwa H and Ueda Y. Intraosseous benign notochordal cell tumors: overlooked precursors of classic chordomas? Histopathology 2004; 44: 597-602.

41. Foote RF, Ablin G and Hall W. Chordoma in siblings. California Med 1958; 88; 383-386.

42. Enim IP. Khordoma nosoglotki u dvukh odnoi sem'I (Chordoma of the nasopharynx in two members of the same family). Westn Otorinolaringol 1963; 26: 88-90.

43. Kerr WA, Haynes DR and Sellars SL. Familial nasopharyngeal chordoma. S Afr Med 1975; 49: 1584.

44. Chetty R, Levin CV and Kalan MR. Chordoma: a 20-year clinicopathological review of the experience at Groote Schuur Hospital, Cape Town. J Surg Oncol 1991; 46: 261-264.

45. Korczak, JF, Kelley MJ, Allikian KA, Shah AA, Goldstein AM and Parry DM. Genomic screening for linkage in a family with autosomal dominant chordoma. Am J Hum Genet 1997; 61: A400.

46. Riva P, Crosti F, Orzan F, Dalprà L, Mortini P, Parafioriti A, Pollo B, Fuhrman Conti AM, Miozzo M and Larizza L. Mapping of candidate region for chordoma development to $1 \mathrm{p} 36.13$ by LOH analysis. Int J Cancer 2003; 107: 493-497.

47. Sawyers JR, Husain M and Al-Mefty $O$. Identification of isochromosome $\mathrm{lq}$ as a recurring chromosome aberration in skull base chordomas: a new marker for aggressive tumors? Neurosurg Focus 2001; 10: 1-6.

48. Scheil S, Brüderlein S, Liehr T, Starke H, Herms J, Schulte M and Möller P. Genome-wide analysis of sixteen chordomas by comparative genomic hybridization and cytogenetics of the first human chordoma cell line, U-CH1. Genes Chromosomes Cancer 2001 ; 32: 203-211.

49. Van Slegtenhorst M, Nellist M, Nagelkerken B, Cheadle J, Snell $R$, van den Ouweland A, Reuser A, Sampson J, Halley D and van der Sluijs P. Interaction between hamartin and tuberin, the TSC 1 and TSC2 gene products. Hum Mol Genet 1998; 7: 1053-1057. 
50. Hodges AK, Li S, Maynard J, Parry L, Braverman R, Cheadle JP, DeClue JE and Sampson JR. Pathological mutations in TSC 1 and TSC2 disrupt the interaction between hamartin and tuberin Hum Mol Genet 2001; 10: 2899-2905

51. Nellist M, Verhaaf B, Goedbloed MA, Reuser AJ, van den Ouweland AM and Halley DJ. TSC2 missense mutations inhibit tuberin phosphorylation and prevent formation of the tuberin-hamartin complex. Hum Mol Genet 2001; 10: 2889 2898.

52. Dutton RV and Singleton EB. Tuberous sclerosis: a case report with aortic aneurysm and unusual rib changes. Pediatr Radiol 1975; 3: 184-186.

53. Schroeder BA, Wells RG, Starshak RJ and Sty JR. Clivus chordoma in a child with tuberous sclerosis: $C T$ and $M R$ demonstration. J Comput Assist Tomogr 1987; 11: 195-196.

54. Börgel J, Olschewski H, Reiter t, Miterski B and Epplen JT. Does the tuberous sclerosis complex include clivus chordoma? A case report. Eur J Pediatr 2001; 160: 138.

55. Sandberg AA and Bridge JA. Chordoma 1995. In: The Cytogenetics of Bone and Soft Tissue Tumors. Sandberg AA, Bridge JA (editors). RG Landers Co., Austin, pp. 251-255.

56. Butler MG, Dahir GA, Hedges LK, Juliao SF, Sciadini MF and Schwartz HS. Cytogenetic, telomere and telomerase studies in five surgically managed lumbosacral chordomas. Cancer Genet Cytogenet 1995; 85: 51-57.

57. Persons DL, Bridge JA and Neff JR. Cytogenetic analysis of two sacral chordomas. Cancer Genet Cytogenet 1991; 56: 197-201.

58. Kuzniacka A, Mertens F, Strombeck B, Wiegant J and Mandahl N. Combined binary ratio labeling fluorescence in situ hybridization analysis of chordoma. Cancer Genet Cytogenet 2004; 151: 178-181.

59. Gil Z, Fliss DM, Voskoboinik N, Leider-Trejo L, Spektor S, Yaron $\mathrm{Y}$ and Orr-Utreger A. Cytogenetic analysis of three variants of clival chordoma. Cancer Genet Cytogenet 2004; 154: 124-130.

60. Gibas Z, Miettinen M and Sabdberg AA. Chromosomal abnormalities in two chordomas. Cancer Genet Cytogenet 1992; 58: 169-173.

61. Chadduk WM, Boop FA and Sawyers JR. Cytogenetic studies of pediatric brain and spinal cord tumors. Pediatr Neurosurg 1992; 17: 57-65.

62. Mertens F, Kreicbergs A, Rydholm A, Willén H, Carlén B, Mitelman F and Mandhal N. Clonal chromosome aberrations in three sacral chordomas. Cancer Genet Cytogenet 1994; 73: 147-151.

63. Buonamici L, Roncaroli F, Fioravanti A, Losi L, Van den Berghe $H$, Calbucci F and Dal Cin P. Cytogenetic investigation of chordomas of the skull. Cancer Genet Cytogenet 1998; 1 12: 49-52.

64. Tallini G, Dorfman H, Brys P, Dal Cin P, De Wever I, Fletcher CDM, Jonson K, Mandahl N, Mertens F, Mitelman F, Rosai J, Rydholm A, Samson I, Sciot R, Van den Berghe H, Vanni R and Willén $\mathrm{H}$. Correlation between clinicopathological features and karyotype in 100 cartilaginous and chordoid tumors. A report from the Chromosome and Morphology (CHAMP) Collaborative Study Group. J Path 2002; 196: 194-203.

65. Brandal P, Bierkehagen B, Danielsen $H$ and Heim S Chromosome 7 abnormalities are common in chordomas. Abstracts of the 9th European Workshop on Cytogenetics and Molecular Genetics of Human Solid Tumors, Brno, Czech Republic; September 16-19, 2004, p 52.

66. Hruban RH, Traganos F, Reuter VE and Huvos AG. A DNA flow cytometric and immunohistochemical study with histogenetic implications. Am J Pathol 1990; 137: 435-447.

67. Ross AJ, Ruiz-Perez V, Wang Y, Hagan DM, Scherer S, Lynch SA, Lindsay S, Custard E, Belloni E, Wilson DI, Wadey R, Goodman
F, Ostarsvik KH, Monclair T, Robison S, Reardon W, Burn J, Scambler P and Strachan T. A homeobox gene, HLXB9, is the major locus for dominantly inherited sacral agenesis. Nat Genet 1998; 20: 358-361.

68. Eisenberg MB, Woloschak M, Sen C and Wolfe D. Loss of heterozygosity in the retinoblastoma tumor suppressor gene in skull base chordomas and chondrosarcomas. Surg Neurol 1997; 47: 156-160.

69. Klingler L, Shooks J, Fiedler PN, Marney A, Butler MG and Schwartz HS. Microsatellite instability in sacral chordoma. J Surg Oncol 2000; 73: 100-103.

70. Matsuno A, Sasaki T, Nagashima T, Matsuura R, Tanaka H, Hirakawa M, Murakami M and Kirino T. Immunohistochemical examination of proliferative potentials and the expression of cell cycle-related proteins of intracranial chordomas. Hum Pathol 1997; 28: 714-719.

71. Ragnarsson G, Eiriksdottir G, Johannsdottir JT, Jonasson JG, Egilsson $V$ and Ingvarsson $S$. Loss of heterozygosity at chromosome $1 \mathrm{p}$ in different solid human tumours: association with survival. Br J Cancer 1999; 79: 1468-1474.

72. Maris JM, Guo C, Blake D, White PS, Hogarty MD, Thompson PM, Rajalingam V, Gerbing R, Stram DO, Matthay KK, Seeger RC and Brodeur GM. Comprehensive analysis of chromosome $1 p$ deletions in neuroblastoma. Med Pediatr Oncol 2000; 36: 32-36.

73. Gastman BR, Johnson DE, Whiteside TL and Rabinowich $\mathrm{H}$. Tumor-induced apoptosis of T lymphocytes: elucidation of intracellular apoptotic events. Blood 2000 15; 95: 2015-2023.

74. Naruse-Nakajima C, Asano M and Iwakura Y. Involvement of EphA2 in the formation of the tail notochord via interaction with ephrinA1. Mech Dev 2001; 102: 95-105.

75. Ogawa Y, Nishioka A, Inomata T, Kataoka S, Nakayama K, Kariya S, Terashima M, Kubonishi I and Yoshida S. lonizing radiation-induced apoptosis in human lymphoma cell lines differing in p53 status. Int J Mol Med 2000; 5: 139-143.

76. Wehr R and Gruss P. Pax and vertebrate development. Int J Dev Biol 1996; 40: 369-377.

77. Ozaki T, Nakamura Y, Enomoto H, Hirose M and Sakiyama S. Overexpression of DAN gene product in normal rat fibroblasts causes a retardation of the entry into the $S$ phase. Cancer Res 1995; 55: 895-900

78. Pizzuti A, Amati F, Calabrese G, Mari A, Colosimo A, Silani V, Giardino L, Ratti A, Pesso D, Calza L, Palka G, Scarlato G, Novelli $G$ and Dallapiccola B. cDNA characterization and chromosomal mapping of two human homologues of the Drosophila dishevelled polarity genes. Hum Mol Genet 1996; 5: 953-958.

79. Momose S, Kobayashi T, Mitani H, Hirabayashi M, Ito K, Ueda M, Nabeshima $Y$ and Hino $O$. Identification of the coding sequences responsible for Tsc2-mediated tumor suppression using a transgenic rat system. Hum Mol Genet 2002 (15); 11: 2997-3006.

80. Tapon N, Ito N, Dickson BJ, Treisman JE and Hariharan IK. The Drosophila tuberous sclerosis complex gene homologs restrict cell growth and cell proliferation. Cell 2001; 105: 345-355.

81. Potter CJ, Huang $H$ and $X u$ T. Drosophila Tscl functions with Tsc2 to antagonize insulin signaling in regulating cell growth, cell proliferation, and organ size. Cell 2001; 105: 357-368.

82. Hengstschlager M, Rodman DM, Miloloza A, HengstschlagerOttnad E, Rosner M and Kubista M. Tuberous sclerosis gene products in proliferation control. Mutat Res 2001; 488: 233-239.

83. Li Y, Corradetti MN, Inoki K and Guan KL. TSC2: filling the GAP in the mTOR signaling pathway. Trends Biochem Sci 2004; 29: 32-38.

84. Sassin JF and Chutorian AM. Intracranial chordoma in children. Arch Neurol 1967; 17: 89-92. 
85. Maiorano E, Renzulli G, Favia G and Ricco R. Expression of intermediate filaments in chordomas. An immunocytochemical study of five cases. Pathol Res Pract 1992; 188: 901-907.

86. Naka T, Iwamoto Y, Shinohara N, Chuman H, Fukui M and Tsuneyoshi M. Cytokeratin subtyping in chordomas and the fetal notochord: an immunohistochemical analysis of aberrant expression. Mod Pathol 1997; 10: 545-551.

87. Gotz W, Kasper M, Miosge N and Hughes RC. Detection and distribution of the carbohydrate binding protein galectin-3 in human notochord, intervertebral disc and chordoma. Differentiation 1997; 62: 149-157.

88. Juliao SF, Rand N and Schwartz HS. Galectin-3: a biologic marker and diagnostic aid for chordoma. Clin Orthop 2002; 397: 70-75.

89. Naka T, Oda Y, Shinohara N, Chuman H, Fukui M and Tsuneyoshi M. Immunohistochemical analysis of E-cadherin, alpha-catenin, beta-catenin, gamma-catenin, and neural cell adhesion molecule (NCAM) in chordoma. J Clin Pathol 2001; 54: 945-950.

90. Horiguchi H, Sano T, Quian ZR, Horokawa M, Kagawa N, Yamaguchi T, Hirose T and Nagahiro S. Expression of cell adhesion molecules in chordomas: an immunohistochemical study of 16 cases. Acta Neuropathol 2004; 107: 91-96.

91. Camacho-Arroyo I, Gonzalez-Aguero G, Gamboa-Dominguez A, Cerbon MA and Ondarza R. Progesterone receptor isoforms expression pattern in human chordomas. J Neurooncol 2000; 49: 1-7.

92. Deniz ML, Kilic T, Almaata I, Kurtkaya O, Sav A and Pamir MN. Expression of growth factors and structural proteins in chordomas: basic fibroblast growth factor, transforming growth factor alpha, and fibronectin are correlated with recurrence. Neurosurgery 2002; 51: 753-760.

93. Druker BJ. STI571 (Gleevec TM) as a paradigm for cancer therapy. Trends Mol Med 2002; 8 (Suppl): S14-18.

94. Pietras K, Sjöblom T, Rubin K, Heldin CH and Östman A. PDGF receptors as cancer drug targets. Cancer Cell 2003; 3: 439-443.

95. Casali PG, Messina M, Stacchiotti S, Tamborini E, Crippa F, Gronchi A, Orlandi R, Ripamonti C, Spreafico C, Bertieri R, Bertulli R, Colecchia M, Fumagalli E, Greco A, Grosso F, Olmi P, Pierotti MA and Pilotti S. Imatinib mesylate in chordoma. Cancer 2004; 101: 2086-2097. 